\title{
Is it the world or is it me!?
}

\author{
S. C. Barclay ${ }^{1}$
}

Key points

Asks whether we as a profession are being responsible enough in our everyday practice to minimise risk to our patients.
Questions how we should seek to arrive at a professional consensus on what might reasonably be regarded as dental 'never events'.
Asks whether there is more we can do to address the so-called 'no-blame culture' to which we all aspire, but may not truly exist within healthcare in the UK at the present time.

Since 2015 the body known as NHS Improvement has published, and this year updated, its list of never events - defined as 'serious incidents that are entirely preventable because guidance or safety recommendations providing strong systemic protective barriers are available at a national level.' How this is interpreted, however, especially in dental terms, has been very poorly managed in my opinion, leading to the potential for increased risk of such events happening and raising anxiety levels among many colleagues, especially the newly qualified, and responses to them both at a local and national level potentially give the lie to the no-blame culture purported to exist within healthcare in the UK. This paper is intended to stimulate debate among those who lead our profession towards a realistic consensus view on management of these issues for the safety of our patients and the peace of mind of the profession.

\section{Is it the world or is it me!?}

As a senior hospital dental consultant in my last handful of years in the profession I look around me increasingly and ask that question. I have done it again, in response to the most recent edict on so-called 'never events', ${ }^{1,2}$ especially as some appear to wish to apply them to dentistry.

Now do not get me wrong, I am most certainly not one to let slipshod clinical or administrative work within the busy world of the NHS go unchallenged, but I do get disheartened at the apparent 'dumbing down' of what was drilled into me as a dental undergraduate and junior trainee over 30 years ago as 'professional care and responsibility'.

The latest information circulated earlier this year from NHS Improvement in relation to never events, just seems again to overcomplicate matters as some would wish to apply it to the world of dentistry as well as in

Consultant in Restorative Dentistry, Newcastle Dental Hospital, Richardson Road, Newcastle upon Tyne, NE2 4AZ Correspondence to: Stewart Barclay

Email: Stewart.Barclay@nuth.nhs.uk

Refereed Paper. Accepted 3 May 2018

DOI: $10.1038 /$ sj.bdj.2018.533 wider healthcare. However, it does seem to be much clearer on a fact I sought unsuccessfully to challenge locally at the last iteration, namely that wrong site local analgesia injections should not be included per se as a recordable and serious never-event. There seems to be (from my reading of the documentation) further clarification of the 'block' argument we had before. This appears to confirm the view held by some of us that this is intended to relate to nerve ablation treatment designed to give long-term pain relief effects as an alternative to surgery, not short-term local analgesia for provision of another treatment.

I would agree that a wrong site application of local anaesthetic has the potential to increase the likelihood of going on to undertake an incorrect side procedure. However, surely a fixation on the delivery of local anaesthetic, leading to a near miss event, rather than the tooth or procedure for which it is being administered, 'blocks' one's view of that more definitive aspect of the process which is much more likely to define the correct location for treatment? Undoubtedly, we should seek to learn from any such errors and the current concepts of 'no harm incidents' as well as 'near misses' should be seen as important opportunities to learn and improve, but I am not clear just how many wrong site inferior dental blocks in themselves are likely to have led on ultimately to a wrong site extraction or surgical procedure.

Interestingly, elsewhere in the new guidance, I could take the opposite view, however, as I am not at all convinced by the argument regarding not recording a wrong primary tooth extraction under a general anaesthetic as wrong site surgery. The guidance suggests that such an error has potentially no long-term consequences - really? - in a child with no permanent successor present for the incorrectly extracted primary tooth (as happens not entirely infrequently) that has significant consequences regardless of being under LA or GA! I cannot see the logic behind the acceptability of this suggestion at all. A wrong tooth extraction has potential consequences for any patient and is perhaps the most important dental "Never Event", if not necessarily in terms of overall medical harm, but in terms of the impact it might have on the patient and their future dental treatment requirements.

With respect to the definition of 'alteration of anatomy' and its potential for inclusion in dental cases, from a dental perspective that is 
a hugely grey area - does that mean that every little unintended and clinically insignificant bump of a bur on an adjacent tooth by a training student (or even an experienced clinician on a very odd occasion!) should be reported and counted as a 'never event' - I would hope not. Equally in a tooth already restored, contact of the bur with an existing restoration does not alter anatomy, does it (as it is already altered)? Also, in surgical terms, does it mean every time a carious or heavily restored tooth adjacent to one being extracted loses a small amount of tooth tissue during the procedure because it is weakened already, does that count too, or if a small amount of enamel is lost at the time of removal of a fixed orthodontic appliance? These are all risks which should be expressed to the patient/carer as part of the consent process, but not necessarily used to clog up incident reporting systems as 'never events'.

I think we are in grave danger of overthinking and over-reporting issues of minimal consequence to our patients if we think we should report all these matters - with the added consequence that we can then expect huge criticism unnecessarily (as the 'no blame culture' clearly still does not really exist in healthcare provision as most will have seen in the recent Bawa-Garba case!).

Perhaps we need to look at the definition in the primary document ${ }^{1}$ for a realistic approach: 'Never events are defined as Serious Incidents that are wholly preventable because guidance or safety recommendations that provide strong systemic protective barriers are available at a national level and should have been implemented by all healthcare providers
... each Never Event type has the potential to cause serious patient harm or death." Should we therefore take a more pragmatic approach as to what is the actual level of serious harm to our patients in terms of what we record as never events.

Interestingly, we in the hospital services are presently expected to work to this NHS Improvement guidance, despite the fact that it clearly does not really address issues in dentistry to any great extent. Some work has been done on never events in primary care dentistry, with a recent paper published in this journal ${ }^{3}$ since the preliminary draft of this paper was submitted, and a previous paper ${ }^{4}$, both addressing candidate never events for primary care dentistry, which are, in my opinion, broadly transferable to dentistry as practised in all environments in the UK.

The paper by Ensaldo-Carrasco et al. ${ }^{3}$, refers to a range of 'patient safety checklists' (derived from human factors research relating to a number of dental procedures), and clearly has gone to some lengths to identify potential dental 'never events'. Most are indisputable, but I think it would be immensely challenging to completely avoid, for example the occasional risk of escape of hypochlorite outwith the confines of the root canal space, even by following guidance fully. We need to recognise that some events are unavoidable accidents, such that designating them as true 'never events' is unrealistic and potentially unhelpful. We should, however, seek to learn from such accidents whenever they occur.

I reiterate that I absolutely agree that we should all do our level best, every time we treat a patient, to avoid things which, with a modicum of professional care and attention are avoidable. In my view, if we allow ourselves to be dragged too far down this route we will be putting in so many artificial checks and balances that we will risk creating a greater likelihood of errors creeping in. In addition, we will be training a new generation of colleagues for whom it is no longer a professional expectation to think first before doing anything to a patient, but automatons for whom it is no more than an artificial, statutory, time consuming obligation to do so each and every time they seek to treat a patient, and remove from them that level of professional responsibility we should carry with us each and every day.

What concerns me further is that I have seen virtually nothing thus far from our professional representatives on this, either the BDA or dental faculties of the Royal Colleges, or for those of us working in teaching hospitals, the Council of Dental Deans or Association of Dental Hospitals, in terms of a nationally agreed view on how this NHS Improvement advice should be translated into the world of dentistry. Hopefully this paper will stimulate further prompt discussion on the issue.

1. NHS Improvement. Never Events policy and framework. 2018. Available at https: //improvement.nhs.uk/ resources/never-events-policy-and-framework/ (accessed June 2018).

2. NHS Improvement. Never Events list 2018. 2018. Available at https://improvement.nhs.uk/documents/2266/Never Events_list_2018_FINAL_v5.pdf (accessed June 2018).

3. Ensaldo-Carrasco E, Carson-Stevens A, Cresswell K, Bedi $R$, Sheikh $A$. Developing agreement on never events in primary care dentistry: an international eDelphi study. $\mathrm{Br}$ Dent J 2018; 224: 733-740.

4. Black I, Bowie P. Patient safety in dentistry: development of a candidate 'never event' list for primary care. Br Dent J 2017; 222: 782-788. 